\title{
Melanosis of the vulva: A long-term follow-up from Brisbane, Australia
}

\author{
Ian S. C. Jones
}

Women’s and Newborn Services, Royal Brisbane and Women’s Hospital, Brisbane and University of Queensland, Herston, Australia Email: ian_jones@health.qld.gov.au

Received 14 May 2012; revised 21 June 2012; accepted 4 July 2012

\begin{abstract}
Object: To determine if vulvar melanosis progressed to melanoma over a period of 20 years or more. Methods: In 2010 the hospital records from the Royal Brisbane Hospital Vulvar Clinic between 1976 and 1988 were reviewed and cross checked with the state wide Queensland Centre for Gynaecological Cancer (QCGC) data base to determine if any patient had been lost to follow up and subsequently developed a vulvar melanoma. Data collected were stored and analysed using the computer software Statistical Package for the Social Sciences (SPSS) 11.0. Results: None of the 12 patients developed vulval melanoma in the years up to 2010. Conclusion: In this small group, followed for more than 20 years, melanosis was not a precursor of melanoma. One patient, who attended the Vulvar Clinic but was not included in this melanosis study, was found to have co-existing melanosis well away from her melanoma in situ and malignant melanoma at presentation. It was not possible to determine if these findings represented a progression of the benign to malignant. Biopsy of abnormal hyper pigmented vulvar skin is recommended. Current knowledge suggests that vulvar melanosis is a benign condition but to be on the safe side follow up of all hyper pigmented vulval lesions to detect early malignant change is recommended.
\end{abstract}

Keywords: Vulva; Pigmented Epithelial Disorders; Features; Follow-Up; Malignancy

\section{INTRODUCTION}

Variations in melanin pigmentation of the vulval skin are considerable within and between different races, age and in relation to hormonal status [1]. Causes of hyperpigmentation due to melanin excess include lentigo; melanin incontinence with melanin within macrophages in the upper dermis following inflammation; adult vulval acanthosis nigricans which affects the entire vulva; pseudo- acanthosis nigricans of flexural skin in obese and darkish skinned people and following fixed drug eruption. The main concern is distinguishing between benign lesions and malignant melanomas of the vulva. Vulval skin biopsy will distinguish between areas of hyperpigmentation due to melanin, melanoma in situ and malignant melanomas. The macroscopic and histological features of normal vulval skin during life have been described elsewhere [2].

The aim of this paper is to review the clinical features, look for predisposing factors and determine the long term outcomes for women who presented to the Vulval Disease Clinic at the Royal Brisbane Hospital between 1976 and 1988 with vulvar melanosis and determine if any lesion progressed to a melanoma.

\section{METHODS}

All patients presenting to the Vulval Diseases Clinic between 1976 and 1988 had their clinical and pathology details recorded on proforma cards in addition to the hospital records. In 2010 the hospital records were reviewed and the proforma cards up dated. Records were cross checked with the state wide Queensland Centre for Gynaecological Cancer (QCGC) data base to determine if any patient had been lost to follow up and subsequently developed a gynaecological malignancy, especially vulval malignant melanoma.

Data from the up-dated proforma cards were stored and analysed using the computer software Statistical Package for the Social Sciences (SPSS) 11.0.

Ethics approval for the review of case records was obtained from the Clinical Research Ethics Committee of the Royal Brisbane and Women’s Hospital.

\section{RESULTS}

Of 361 patients seen at the clinic between 1976 and 1988, 12 (all Caucasian) were found to have vulvar melanosis. Patient age at presentation is shown in Table 1, with the majority being aged 50 or more. The three patients under 50 years of age were all menstruating regularly. All but 
Table 1. Age at time of diagnosis.

\begin{tabular}{ccc}
\hline Age range & $\begin{array}{c}\text { Melanosis } \\
\mathrm{n}=12\end{array}$ & $\begin{array}{c}\text { Melanoma } \\
\mathrm{n}=42\end{array}$ \\
\hline $20-29$ & 0 & 1 \\
$30-39$ & 1 & 0 \\
$40-49$ & 3 & 8 \\
$50-59$ & 2 & 7 \\
$60-69$ & 3 & 6 \\
$70-79$ & 1 & 11 \\
$80-89$ & 2 & 8 \\
$90-99$ & 0 & 1 \\
\hline
\end{tabular}

one patient were parous. Three patients presented with a symptomatic vulvar dystrophy (history of pruritus vulvae for between 3 months and 5 years), one patient presented with pruritus vulvae due to vulval intra epithelial neoplasia (VIN 3) remote from her vulvar melanosis, and one presented with a vaginal discharge. The other patients were referred for review of their vulval pigmentation noted at routine gynaecological examination. The highest incidence of melanosis was found in post menopausal patients (67\%), but can occur before this time.

The anatomical location of the vulvar melanosis appeared to "favour" the labia minora (8 cases) and introitus ( 2 cases). The lesions were of variable size $(1-5 \mathrm{~cm})$, flat and the affected skin had a normal texture. No patients had clinical groin lymphadenopathy. In an attempt to determine if there were any predisposing features for the development of vulvar melanosis coexisting medical conditions were sought. Various conditions were found in 11 patients (Table 2), but apart from the possibility of skin irritation associated with the presence of a vulvar dystrophy being a cause rather than an association, no predisposing features were found.

No treatments were used to manage the vulvar melanosis and no patients developed malignant melanoma of the vulva between 25 and 33 years after presenting to the Vulval Diseases Clinic. However, another patient aged 75 who attended the Vulvar Clinic but not included in this melanosis study, was found to have co-existing melanosis well away from her melanoma in situ and malignant melanoma at presentation in 1977.

QCGC data from the beginning of data collection in 1985 to 2009 record 42 patients with vulval melanoma of whom 20 died of their disease and 6 died of unrelated causes. Their ages at diagnosis ranged from 25 to 92 years and this data was compared with the ages of diagnosis for the melanosis cases (Table 1). Treatment regimes for the melanoma group included wide local excision; partial vulvectomy; radical vulvectomy and bilateral
Table 2. Other medical disorders associated with non-neoplastic epithelial disorders.

\begin{tabular}{cc}
\hline Patient identifier & Other medical conditions present \\
\hline 1 & Liver disease, HBP \\
2 & $\begin{array}{c}\text { Vulvar lichen sclerosus, non-Hodgkins } \\
\text { lymphoma, endometrial carcinoma stage 3B }\end{array}$ \\
3 & Micro-invasive cervical carcinoma \\
4 & Diabetes, renal calculi, VIN 3 \\
5 & Vulvar lichen sclerosus, pernicious anaemia, \\
6 & depression \\
7 & BCC nose, renal calculi, HBP \\
8 & Cervical carcinoma with radiotherapy treatment \\
9 & Gout, brother with melanoma \\
10 & Nil recorded \\
11 & BCC scalp, scleroderma, anxiety \\
12 & BCC ear \\
\hline
\end{tabular}

groin dissection; radical vulvectomy, vaginectomy and groin dissection; pelvic lymphadenectomy and surgery plus radiation therapy.

\section{DISCUSSION}

The aims of this paper were to determine if vulvar melanosis progressed to melanoma over a period of 20 years or more; review the clinical features; determine if there were any predisposing features for the development of melanoma and determine the long term outcomes for women who presented to the Vulvar Disease Clinic with vulvar melanosis. The need for a long term follow-up study of melanosis was because it is unknown if melanosis could be a forerunner of melanoma, and if it was how long such changes take to occur. Table 1 shows the age distribution for patients presenting with vulval melanosis (36 to 85 years) compared with vulval melanoma (25 to 92 years), which because of advanced age when discovered suggests some patients take a considerable time for a melanoma to develop. Even with a 30 year follow up this may not be long enough for some patients to develop vulvar melanoma.

The prevalence of vulval melanosis is unknown. Large melanotic lesions of the vulva are uncommon [3,4]. A study of 301 new patients presenting to a gynaecology practice over a one year period found 31 (10\%) had pigmented lesions on the vulva, six (2\%) had diffuse hyper pigmentation of the vulva and seven (2\%) had vulval nevocytic nevi [5].

The most common cause of vulval hyperpigmentation due to melanin (which must be distinguished histolo- 
gically from haemosiderin), is lentigo [1]. Lentigo, also known as lentigo simplex, is a benign pigmented prolixferation of epidermal or mucous membrane melanocytes which form smooth, non-infiltrating dark brown lesions on the labia minora and introitus. Lentigo is of unknown aetiology and pathogenesis and lesions do not usually exceed one $\mathrm{cm}$ in diameter [1]. However, in the current study melanosis varied in size from one to five $\mathrm{cm}$. Sison-Torre \& Ackerman [6] described eight women with extensive pigmentation of the vulva and used the term melanosis of the vulva. They showed on biopsy that these lesions were benign and analogous to lentigo. Lentigines may resemble junctional naevi, but do not exhibit cytological atypia [7]. This raises the question could vulvar melanosis be the big sister to lentigo simplex with the difference being size alone?

Vulval pigmentation depends on the presence of cells containing melanin [8]. The detection of such pigmentation can be obscured by dermal vascularity, the thickness of overlying skin and the position of the melanin containing cells within the skin. The distribution of pigmentation of dark-skinned races is concentrated in the basal layer, but varies considerably in amount. Microscopic patches of melanin pigment were found in almost 80 per cent of 35 Caucasian women at post mortem when there was no evidence of macroscopic hyper pigmentation [8].

The differential diagnosis of vulval hyperpigmentation is lentigo; junctional naevi; following inflammatory conditions like lichen planus; adult vulval acanthosis nigricans which affect the entire vulva and may be associated with an adenocarcinoma or occasionally lymphoma or epithelial carcinoma; the pseudo-acanthosis nigricans of flexural skin in obese and darkish skinned people (now thought to be related to insulin resistance) and following fixed drug eruption from for example, sulphonamides. The most important risk for these patients is the possibility of misdiagnosing a malignant melanoma. Making a clinical diagnosis based on the anatomical site and skin texture is not foolproof, hence biopsy and histological diagnosis is recommended. Even in the case of the 75 year old woman who presented with co-existing melanosis, melanoma in situ and malignant melanoma, it was not possible to determine if these findings represented a progression from the benign to malignant. The importance of follow up of all hyper pigmented vulval lesions to detect early malignant change is stressed.

Variations in terminology to describe benign hyper- pigmentation due to the presence of melanin include vulvar melanosis, idiopathic lenticular mucocutaneous pigmentation and genital lentiginosis [1]. However vulvar melanosis or its other name melanosis vulvae seem to be terms used by the majority when referring to this condition.

\section{CONCLUSION}

The difficulty in finding a suitable term for benign hyperpigmentation of vulval skin conditions remains. To reliably diagnose a hyper pigmented vulval lesion a histopathology diagnosis is required. Current knowledge suggests that vulvar melanosis is a benign condition but to be on the safe side follow up of all hyper pigmented vulval lesions to detect early malignant change is recommended.

\section{ACKNOWLEDGEMENTS}

Thanks to the Queensland Centre for Gynaecological Cancer (QCGC) staff for their assistance in providing data and checking their data on the current study patients.

\section{REFERENCES}

[1] Neill, S.M. and Lewis, F.M. (2009) Ridley's the vulva. 3rd Edition, Wiley-Blackwell, Hoboken.

[2] Jones, I.S.C. (1983) A histological assessment of normal vulval skin. Clinical and Experimental Dermatology, 8, 130-138. doi:10.1111/j.1365-2230.1983.tb01818.x

[3] Estrada, R. and Kaufman, R. (1993) Benign vulvar melanosis. The Journal of Reproductive Medicine, 38, 5-8.

[4] Barnhill, R., Albert, L., Shama, S., Goldenhersh, M., Rhodes, A. and Sober, A. (1990) Genital lentiginosis: A clinical and histopathic study. Journal of the American Academy of Dermatology, 22, 453-460. doi:10.1016/0190-9622(90)70064-O

[5] Rock, B., Hood, A. and Rock, J. (1990) Prospective study of vulvar nevi. Journal of the American Academy of Dermatology, 22, 104-106. doi:10.1016/0190-9622(90)70017-C

[6] Sison-Torre, E.Q. and Ackerman, A.B. (1985) A clinical simulator of malignant melanoma: Melanosis of the vulva. The American Journal of Dermatopathology, 7, 51-60. doi:10.1097/00000372-198501001-00013

[7] Janovski, N.A. and Douglas, C.P. (1972) Diseases of the vulva. Harper \& Row Publishers, Hagerstown.

[8] Jones, I.S.C. (1979) An Assessment of vulval pigmentation. The New Zealand Medical Journal, 89, 348-350. 